\title{
Estimating Nigerian Power System Post Contingency Line Flows Using Power Distribution Factors
}

\author{
Emmanuel. A. Anazia ${ }^{1}$, Onyedikachi N. Samuel ${ }^{2}$, Obroh O. Rebecca ${ }^{3}$ \\ ${ }^{1}$ Senior Lecturer, ${ }^{2}$ Lecturer, ${ }^{3}$ M.Eng Student \\ Electrical Engineering Department \\ ${ }^{1,2}$ Nnamdi Azikiwe University Awka, Anambra, Nigeria \\ ${ }^{3}$ International Breweries PLC, Port Harcourt Plant, Rivers, Nigerian
}

\section{ABSTRACT}

At the point of convergence of any power system AC load flow iterative method, the principal bus parameters necessary to compute the transmission line flows and losses are provided. The exactness and accuracy of the result depends largely on the iterative method and the iteration termination criteria and each operating condition requires a unique solution of the analysis. State estimation techniques are also viable alternatives to AC load flow techniques in estimating network bus parameters from a known state but the speed of estimation is comparatively similar to the AC counterpart. However, the justification for the use of DC load flow for quick estimation of transmission line flows as against the $\mathrm{AC}$ is that the resulting mismatch is negligible when used for contingency and security analyses. Estimates of transmission line active power flow can be made using linear distribution/sensitivity factors whose result match those of DC load flow. These sensitivity factors: Power Transfer Distribution Factors (PTDF) and Line Outage Distribution Factors (LODF) are calculated and stored for a network and remains valid if the network remains significantly unmodified. With these stored factor from an operating point, post contingency flows may be predicted on any line. In this work, the PTDF and LODF of the Nigerian $330 \mathrm{kV}$ of 41 bus were computed and stored from a base case, then post contingency flows were predicted for the 77 transmission lines of the network following contingency in the form of 140MW load shedding at bus $1,50 \%$ generator output reduction at bus 2 , 100MW generator output increment at bus 20 and 100MW generator output decrease at bus 25. The result shows that using sensitivity factors to estimate transmission line flow works as validated by the result from load DC load flow technique. Therefore, a quicker, linear and non-iterative method is validated in order to estimate transmission line flows from a known operating point with the slack bus responsible for active power exchanges.

Keyword: Distribution Factors; Post Contingency Flow; Line Outage; Generator Outage; Transmission Lines; Sensitivity Factors; Nigerian Network

\section{CINTRODUCTION}

For secure operation of interconnected power system components, safe operational limits are defined that must not be violated if continued reliable supply is to be sustained. Hence, meeting consumer electricity demand must not be attained at the expense of component safety nor the stability of the entire system. However, the inevitable changing operational conditions sometimes threaten to violate these safe limits. In other words, network complexity and condition of operation can result in system instability/collapse or at the very least forced component outages. It is instructive to note that not all component outage whether forced (as in the case of un-cleared faults) or scheduled (due to maintenance or repairs) lead to violations of prescribed limits on transmission lines or buses. But there are outages of certain components at certain operating condition that result in a significant alteration of the state of the entire power system. For instance, the outage a heavy loaded transmission line connected to a load center means significant widespread blackout at the load center leading to customer dissatisfaction. Then again, the sudden outage of a large generator may lead to undesired voltage profile containing violations across 
a number of nodes which may culminate in voltage collapse. In other words, each components outage has a unique implication which is largely dependent on the component defined limits, its role and the condition of system operation. Therefore, a comprehensive knowledge of the network components and their condition of operation at the levels of design, planning and operation is indispensible for reliable supply and to this end, stability assessment methods are employed. One of such methods is sensitivity analysis which measures the sensitivity of a line component with respect to an outage or variation of flow on another transmission line or from generator at a node.

\section{SENSITIVITY ANALYSIS}

The word sensitivity is defined to be the degree of response of a system, to a change in the input signal. An extended definition with respect to power system network would mean that sensitivity analysis of power grid components is the process of determining the impact or effect on a particular system variable will cause and how it would vary from a known or desired state [1]. This enables the system planner or operator, to determine how the entire system would respond to a change and in this case an outage forced or scheduled. With respect to this, the pertinent questions are: is it possible to estimate a postcontingency power flow on transmission lines? Is it also possible to estimate power system defined limit violations and estimate the margin to violation from a current operational state following a viable grid component contingency? Such capability makes it possible to screen and select credible contingencies from a pool of possible contingencies, rank them on the bases of their severity and/or the number of violations they cause. This screening and ranking would enable remedial or preventive actions to be recommended or implemented to ameliorate the impact of such credible violation.

\section{A. Distribution Factors}

The problem of studying thousands of possible outages becomes herculean if the desire is to present quick results. However, one of the easiest ways to provide a quick calculation of possible overloads is to use linear sensitivity factors [2]. Since generators are the source of real power while transmission lines are the conveyors to the load centers, there is need to ascertain system sensitivities to the flows through these lines, especially when the output of the generator vary within network. Again since generator outputs and line flows of real power are summative, linear analysis involving direct current manipulations seem adequate especially for a lossless network. Linear sensitivity factors are preferred on the account of the ease and speed of calculation of possible overloads especially when studying numerous possible outages [3]. Power distribution factor is about the only technique used in allocating MW flows on the lines for power transaction in the system defines the relative change in the power flow on a particular line due to an injection or withdrawal of power on a pair of buses while line outage distribution factors are linear estimates of the change in flow on adjacent lines with the outage of transmission lines. Basically, they are of two types;

\section{Power Transfer Distribution Factors (PTDF)}

PTDF shows linearized impact of power transfer [4]. It is the relative change in power flow on a particular line due to an injection and withdrawal of power on a pair of buses [5]. They represent the sensitivity of the flow on line $l$ to a shift of power from bus $i$ to bus $k$ [2]. Power Transfer Distribution Factors are also known by other names such as Generation Shift Factors (GSFs), Power Distribution Coefficients (PDCs), Effectiveness Factors and Impedance Factors. The PTDF has four (4) attributes, namely;

$>$ a particular Line (with reference direction)

a particular Bus

$>$ value of the transfer factor

a reference bus

The value of the PTDF of line $l$ with respect to bus $i$ is defined to be the change (or sensitivity) of active megawatt (MW) power flow in a reference direction on line $l$ with respect to a change in injection at bus $i$ and a corresponding change in withdrawal at the reference bus [6]. PTDF has the following mathematical expression as [7]

$$
P T D F_{i, k, l}=\frac{\Delta f_{l}}{\Delta P_{i \text { to } k}}
$$

$P T D F_{i, k, l}=\mathrm{PTDF}$ for power transfer from bus $i$ to bus

$\Delta f_{l}=$ change in line flow of the monitored line when power is transferred

$\Delta P_{i \text { to } k}=$ power transferred from bus $i$ to bus $k$

Numerical range of PTDF includes:

$-1 \leq P T D F_{i, k, l} \leq+1$ 
$P T D F_{i, k, l}=1$, this is an indication that all of the transferred power from $i$ to $k$ must flow through line $l$. If the value is -1 , it means that all of the transferred power from $i$ to $k$ will flow through line $l$, but in a reversed direction and if $P T D F_{i, k, l}=0$, this indicates that none of the power transferred from $i$ to $k$ will pass through line $l[8]$.

\section{Line Outage Distribution Factor (LODF)}

Line outage distribution factors are linear estimates of the change in flow on adjacent lines when transmission lines are lost [9]. They are often applied in checking overloads on the lines following the line loss [10]. The failure/outage of a major transmission line causes redistribution in the line flows and can result in voltage variation within the system. The analysis of transmission line failures requires methods to predict these line flows and voltages.

LODF shows linearized impact of power transfer. They represent the sensitivity of the flow on line $l$ to a line failure in the network [2]. LODFs aids in calculating the impact the opening (outage) of a transmission line will have on all the other lines in the power system. The value of the LODF of line $l$ with respect to loss of line $t$ is defined to be the change (or sensitivity) of active (MW) power flow on line $l$ with line $t$ out.

A simulation program was used to study the cases of line outages in the system using the line outage distribution factors [11]. Outages of lines which cause increased power flow over prescribed limit in the remaining lines of the network are detected. The LODF factors are given as [7];

$$
L O D F_{l, t}=\frac{\Delta f_{l}}{f_{t}^{0}}
$$

$L O D F_{l, t}=$ line outage distribution factor when monitoring line $l$ after an outage on line $t$

$\Delta f_{l}=$ change in MW flow on monitored line $l$

$f_{t}^{0}=$ original flow on outage (open) line $t$

\section{METHODOLOGY}

The aim of this work is to estimate post-contingency on the Nigerian $330 \mathrm{kV}$ transmission line flows using direct current based sensitivity factors. This would be achieved by
1. Estimating the power flow sensitivity of network transmission lines due to generators outages/output variation.

2. Estimating the power flow sensitivity of network transmission lines due to the outage of adjacent transmission lines.

3. Estimating post-outage transmission line active power flows

4. Verifying the post-contingency Active power flows estimated from sensitivity factors using post-contingency DC load-flow results.

The network used for this estimation is the Nigerian $330 \mathrm{KV}$ power system network consisting of 41 buses, 17 generators and 77 transmission lines. The network generator/bus data and line parameters are given in the appendix section.

\section{A. Procedure:}

1. Derive relationships for $\mathrm{AC}$ and $\mathrm{DC}$ load flows

2. Estimate the Transmission line flows for $A C$ and DC methods for the network at a base case.

3. Following any contingency; transmission line outage, reduction/increase or total outage of generation at any generator (beside the Slack bus), estimate the DC line flows for all the transmission lines of the network.

4. Derive the equations representing the sensitivity factors; PTDF and LODF and for the network, evaluate the values of PTDF and LODF.

5. Using PTDF and LODF values, estimate transmission flow for a monitored line with respect to generation reduction, increase or outage and line outage.

6. For a monitored line, compare the estimated flow of (v) to the flows of (iii) above.

Executable MATLAB programs have been used to perform the AC load flow (Newton Raphson iteration), DC load flow, PTDF and LODF estimations.

Predicting Post contingency flow using

\section{B. Mathematical Descriptions}

Direct Current (DC) Load Flow of Sample Network

Unlike the AC load flow, the DC power flow is a noniterative as it simplifies the Fast decoupled AC derivations under certain assumptions. Following these assumptions, the predominant relationship from the Fast decoupled method [12] relates $\Delta P$ and $\Delta \delta$, expressed as 


$$
\begin{gathered}
{\left[\begin{array}{c}
\Delta P_{1} \\
\Delta P_{2} \\
\vdots \\
\Delta P_{(n-1)}
\end{array}\right]=\left[B^{\prime}\right]\left[\begin{array}{c}
\Delta \delta_{1} \\
\Delta \delta_{2} \\
\vdots \\
\Delta \delta_{(n-1)}
\end{array}\right]} \\
{\left[\begin{array}{c}
\frac{\Delta P_{1}}{\left|V_{1}\right|} \\
\frac{\Delta P_{2}}{\left|V_{2}\right|} \\
\vdots \\
\frac{\Delta P_{(n-1)}}{\left|V_{(n-1)}\right|}
\end{array}\right]=\left[\begin{array}{cccc}
B_{11} & B_{12} & \cdots & B_{1(n-1)} \\
B_{21} & B_{22} & \cdots & B_{2(n-1)} \\
\vdots & \vdots & \vdots & \vdots \\
B_{(n-1) 1} & B_{(n-1) 2} & & B_{(n-1)(n-1)}
\end{array}\right]\left[\begin{array}{c}
\Delta \delta_{1} \\
\Delta \delta_{2} \\
\vdots \\
\Delta \delta_{(n-1)}
\end{array}\right]}
\end{gathered}
$$

Where the diagonal and off diagonal of the reactance $\quad x_{i k}=$ reactance of line between bus $i$ and bus $k$ matrix $\mathrm{B}$ is

$B_{i i}=\sum_{k=1}^{N_{b u s}} \frac{1}{x_{i k}} \quad$ and $\quad B_{i k}=B_{k i}=-\frac{1}{x_{i k}} \quad P_{i}=$ Calculated Real power schedule at bus $i$ and $k$

But where $r$, the resistance of the transmission line is significant, then

$B_{i i}=\sum_{k=1}^{N_{b u s}}-B_{i k}$ and $B_{i k}=B_{k i}=-\frac{x_{i k}}{r_{i k}^{2}+x_{i k}^{2}}$

The DC load flow is adequate in estimating approximately accurate MW flows on transformers and transmission lines while ignoring the MVAR and MVA flows. Consequently, form [2], the real or MW power flow on a lossless transmission line connected between bus $i$ and bus $k$ using DC power flow is

$P_{i k}=\frac{1}{x_{i k}}\left(\delta_{i}-\delta_{k}\right)$

\section{$i$ and $k$ represent bus numbers $(1,2,3,4, \ldots, n)$}

Note that $P_{i k}=-P_{k i}$

Then the Power scheduled at bus $i, P_{i}$ is derived using

$$
P_{i}=\sum_{k=1}^{l} P_{i k}
$$

$P_{i k}=$ Real power flowing through transmission line connected between bus $i$ and bus $k$

$\delta_{i} \quad \& \quad \delta_{k}=$ Voltage bus angle at bus $i$ and $k$ respectively

The power network used here is the Nigerian $330 \mathrm{kV}$ interconnected network. This version of the network contains 41 buses, 17 generators and 30 load units which are interconnected by 77 transmission lines. The total system base load is $7491 \mathrm{MW}$ and the generator at bus 27 (Egbin) is used as slack/reference generator. The network oneline diagram is shown below while its network data with respect to bus, generator and line parameters are given in appendix tables $4.13,4.14$ and 4.15 respectively). In order to justify the choice of the DC over the AC flow estimation, we compare the AC and DC MW flows of the network at base case; noting how marginal the mismatch is. 
International Journal of Trend in Scientific Research and Development (IJTSRD) ISSN: 2456-6470

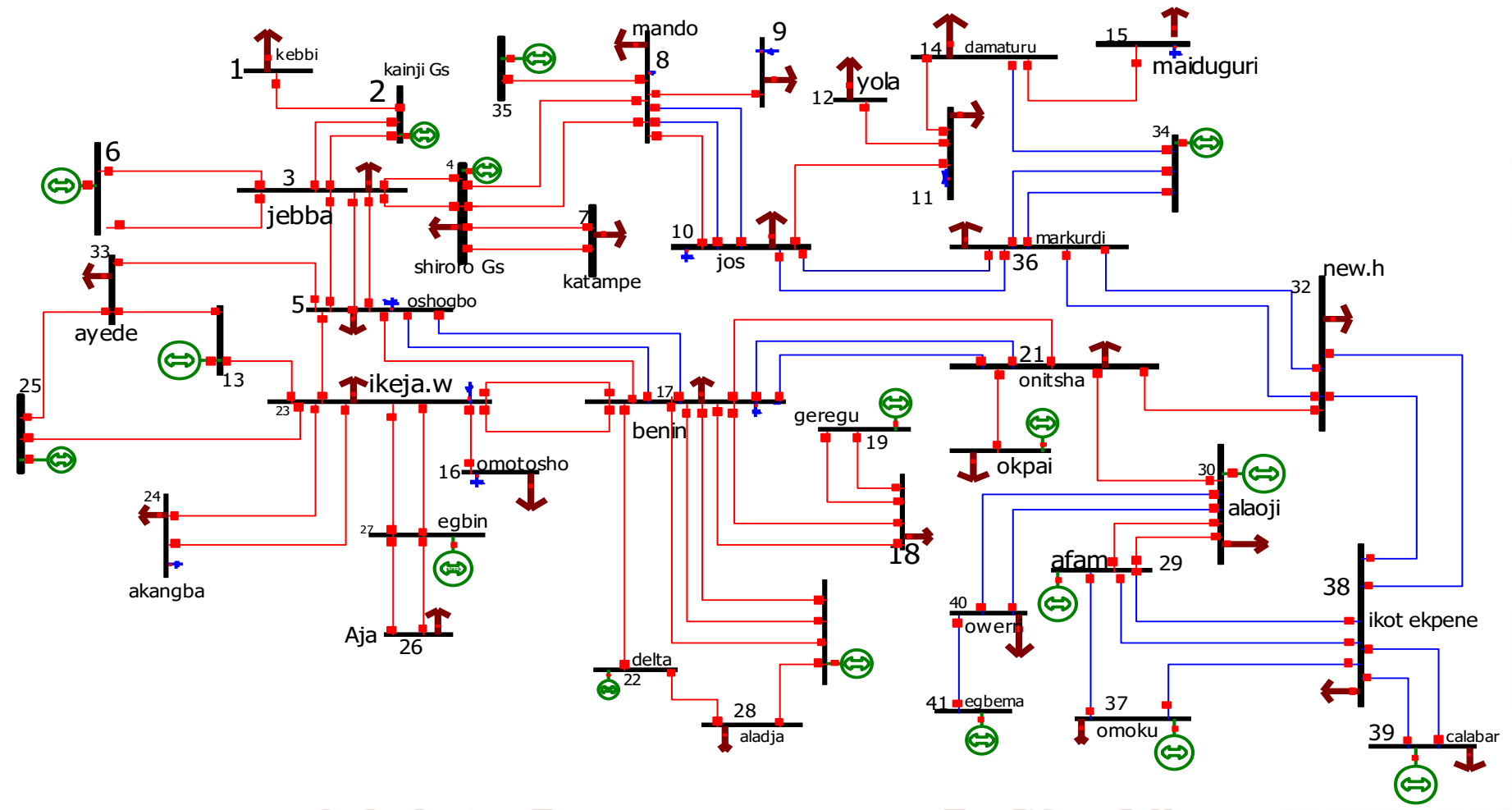

Figure 1: The Nigerian 330kV 41 bus system

Table 1: Base Case MW Flow Mismatch from AC and DC Load Flow Analysis

\begin{tabular}{|c|c|c|c|c|c|c|c|c|c|c|c|}
\hline \multirow{2}{*}{$\begin{array}{c}\text { Line } \\
\text { Cod } \\
\mathrm{e} \\
\end{array}$} & \multirow{2}{*}{$\begin{array}{c}\text { Bus } \\
\text { From- } \\
\text { To } \\
\end{array}$} & \multicolumn{2}{|c|}{ Line MW flow } & \multicolumn{2}{|c|}{ Mismatch } & \multirow{2}{*}{$\begin{array}{c}\text { Line } \\
\text { Cod } \\
\mathrm{e} \\
\end{array}$} & \multirow{2}{*}{$\begin{array}{c}\text { Bus } \\
\text { From- } \\
\text { To } \\
\end{array}$} & \multicolumn{2}{|c|}{ Line MW flow } & \multicolumn{2}{|c|}{ Mismatch } \\
\hline & & $\mathrm{AC}$ & $\mathrm{DC}$ & $\begin{array}{c}\text { Actua } \\
1\end{array}$ & $\%$ & & & $\mathrm{AC}$ & $\mathrm{DC}$ & $\begin{array}{c}\text { Actua } \\
1\end{array}$ & $\%$ \\
\hline L1 & $1-2$ & -150 & -150 & 0 & 0.00 & $\mathrm{~L} 40$ & $18-17$ & 146.93 & 147 & 0.07 & 0.05 \\
\hline L2 & $2-3$ & 223.76 & 225 & 1.24 & 0.55 & $\mathrm{~L} 41$ & $19-18$ & 197 & 197 & 0 & 0.00 \\
\hline L3 & $2-3$ & 223.76 & 225 & 1.24 & 0.552 & L42 & $19-18$ & 197 & 197 & 0 & 0.00 \\
\hline L4 & $3-4$ & 476.38 & 437.91 & 38.47 & 8.08 & L43 & $21-30$ & 74.23 & 57 & 17.23 & 23.21 \\
\hline L5 & $3-4$ & 476.38 & 437.91 & 38.47 & 8.08 & L44 & $21-31$ & $\begin{array}{c}- \\
315.32 \\
\end{array}$ & -320 & 4.68 & 1.48 \\
\hline L6 & $3-5$ & $\begin{array}{c}- \\
119.92 \\
\end{array}$ & -91.94 & 27.98 & 23.33 & L45 & $21-32$ & 552.04 & 504.11 & 47.93 & 8.68 \\
\hline L7 & $3-5$ & $\begin{array}{c}- \\
119.92 \\
\end{array}$ & -91.94 & 27.98 & 23.33 & L46 & $22-28$ & 370.28 & 371.62 & 1.34 & 0.36 \\
\hline L8 & $3-5$ & $\begin{array}{c}- \\
119.92 \\
\end{array}$ & -91.94 & 27.98 & 23.33 & $\mathrm{~L} 47$ & $23-16$ & 305.5 & 300 & 5.5 & 1.80 \\
\hline L9 & $6-3$ & 250 & 250 & 0 & 0.00 & $\mathrm{~L} 48$ & $23-17$ & $\begin{array}{c}- \\
224.26 \\
\end{array}$ & $\begin{array}{c}- \\
281.98 \\
\end{array}$ & 57.72 & 25.74 \\
\hline L10 & $6-3$ & 250 & 250 & 0 & 0.00 & L49 & $23-17$ & $\begin{array}{c}- \\
224.26 \\
\end{array}$ & $\begin{array}{c}- \\
281.98 \\
\end{array}$ & 57.72 & 25.74 \\
\hline L11 & $4-7$ & 177.04 & 175 & 2.04 & 1.15 & L50 & $23-27$ & $\begin{array}{c}- \\
277.23 \\
\end{array}$ & $\begin{array}{c}- \\
176.06 \\
\end{array}$ & $\begin{array}{c}101.1 \\
7 \\
\end{array}$ & 36.49 \\
\hline L12 & $4-7$ & 177.04 & 175 & 2.04 & 1.15 & L51 & $23-27$ & $\begin{array}{c}- \\
277.23 \\
\end{array}$ & $\begin{array}{c}- \\
176.06 \\
\end{array}$ & $\begin{array}{c}101.1 \\
7 \\
\end{array}$ & 36.49 \\
\hline L13 & $4-8$ & 293.88 & 275.41 & 18.47 & 6.28 & L52 & $24-23$ & -235 & -235 & 0 & 0.00 \\
\hline
\end{tabular}


International Journal of Trend in Scientific Research and Development (IJTSRD) ISSN: 2456-6470

\begin{tabular}{|c|c|c|c|c|c|c|c|c|c|c|c|}
\hline L14 & $4-8$ & 293.88 & 275.41 & 18.47 & 6.28 & L53 & $24-23$ & -235 & -235 & 0 & 0.00 \\
\hline L15 & $5-17$ & $\begin{array}{c}- \\
178.22 \\
\end{array}$ & $\begin{array}{c}- \\
174.91 \\
\end{array}$ & 3.31 & 1.86 & L54 & $25-23$ & 72.32 & 95.16 & 22.84 & 31.58 \\
\hline L16 & $5-17$ & $\begin{array}{c}- \\
178.22 \\
\end{array}$ & $\begin{array}{c}- \\
174.91 \\
\end{array}$ & 3.31 & 1.86 & L55 & $27-26$ & 227.86 & 227.5 & 0.36 & 0.16 \\
\hline L17 & $5-17$ & $\begin{array}{c}- \\
178.22 \\
\end{array}$ & $\begin{array}{c}- \\
174.91 \\
\end{array}$ & 3.31 & 1.86 & L56 & $27-26$ & 227.86 & 227.5 & 0.36 & 0.16 \\
\hline L18 & $5-23$ & 11.61 & 45.06 & 33.45 & $\begin{array}{c}288.1 \\
1\end{array}$ & L57 & $28-20$ & 66.7 & 69.62 & 2.92 & 4.38 \\
\hline L19 & $5-33$ & -40.25 & 2.86 & 43.11 & $\begin{array}{c}107.1 \\
1 \\
\end{array}$ & L58 & $29-37$ & 210.34 & 201.74 & 8.6 & 4.09 \\
\hline L20 & $8-9$ & 357.95 & 350 & 7.95 & 2.22 & L59 & $29-38$ & 127.48 & 123.72 & 3.76 & 2.95 \\
\hline $\mathrm{L} 21$ & $8-10$ & 73.9 & 66.94 & 6.96 & 9.42 & L60 & $29-38$ & 127.48 & 123.72 & 3.76 & 2.95 \\
\hline L22 & $8-10$ & 73.9 & 66.94 & 6.96 & 9.42 & L61 & $30-29$ & 7.65 & -0.41 & 8.06 & $\begin{array}{c}105.3 \\
6\end{array}$ \\
\hline L23 & $8-10$ & 73.9 & 66.94 & 6.96 & 9.42 & L62 & 30-29 & 7.65 & -0.41 & 8.06 & $\begin{array}{c}105.3 \\
6\end{array}$ \\
\hline L24 & $10-11$ & 288.68 & 269.38 & 19.3 & 6.69 & L63 & $30-40$ & 49.3 & 49 & 0.3 & 0.61 \\
\hline $\mathrm{L} 25$ & $10-36$ & 159.34 & 159.28 & 0.06 & 0.04 & L64 & $30-40$ & 49.3 & 9 & 0.3 & 0.61 \\
\hline L26 & $10-36$ & 159.34 & $\begin{array}{c}- \\
159.28 \\
\end{array}$ & 0.06 & 0.04 & L65 & $32-38$ & $\begin{array}{c}- \\
285.84 \\
\end{array}$ & 282.09 & 3.75 & 1.31 \\
\hline L27 & $12-11$ & -160 & -160 & $O_{0}$ & 0.00 & L66 & $32-38$ & 285.84 & 282.09 & 3.75 & 1.31 \\
\hline L28 & $13-23$ & 120.69 & 142.7 & 22.01 & 18.24 & L67 & $33-13$ & $\begin{array}{c}- \\
148.85 \\
\end{array}$ & -127.3 & 21.55 & 14.48 \\
\hline L29 & 14-11 & 40.49 & 50.62 & 10.13 & 25.02 & L68 & $33-25$ & -31.38 & -8.84 & 22.54 & 71.83 \\
\hline L30 & $14-15$ & 201.83 & 200 & 1.83 & 0.912 & L69 & $7035-8$ & 200 & 200 & 0 & 0.00 \\
\hline L31 & $14-34$ & $\begin{array}{c}- \\
372.32 \\
\end{array}$ & $\begin{array}{c}- \\
380.62 \\
\end{array}$ & 8.3 & 2.23 & $\mathrm{~L} 70$ & 36-32 & $\begin{array}{c}- \\
444.33 \\
\end{array}$ & $\begin{array}{c}- \\
439.15\end{array}$ & 5.18 & 1.17 \\
\hline L32 & $17-18$ & $\begin{array}{c}- \\
145.35\end{array}$ & & 1.65 & 1.14 & L71 & 36-32 & $\begin{array}{c}- \\
444.33\end{array}$ & $\begin{array}{c}- \\
439.15\end{array}$ & 5.18 & 1.17 \\
\hline L33 & $17-20$ & $\begin{array}{c}- \\
320.01 \\
\end{array}$ & \begin{tabular}{|c|}
- \\
323.21 \\
\end{tabular} & 3. & 1.00 & L72 & $36-34$ & 192.76 & 189.87 & 2.89 & 1.50 \\
\hline L34 & $17-20$ & $\begin{array}{c}- \\
320.01 \\
\end{array}$ & $\begin{array}{c}- \\
323.21 \\
\end{array}$ & 3.2 & 1.00 & L73 & $36-34$ & 192.76 & 189.87 & 2.89 & 1.50 \\
\hline L35 & $17-20$ & $\begin{array}{c}- \\
320.01 \\
\end{array}$ & \begin{tabular}{|c|}
- \\
323.21 \\
\end{tabular} & 3.2 & 1.00 & L74 & $37-38$ & 154.24 & 146.74 & 7.5 & 4.83 \\
\hline L36 & $17-21$ & 142.96 & 118.77 & 24.19 & 16.92 & L75 & $38-39$ & $\begin{array}{c}- \\
154.31 \\
\end{array}$ & -155 & 0.69 & 0.45 \\
\hline L37 & $17-21$ & 142.96 & 118.77 & 24.19 & 16.92 & L76 & $38-39$ & $\begin{array}{c}- \\
154.31 \\
\end{array}$ & -155 & 0.69 & 0.45 \\
\hline L38 & $17-21$ & 142.96 & 118.77 & 24.19 & 16.92 & L77 & $40-41$ & -81.6 & -82 & 0.4 & 0.49 \\
\hline L39 & $17-22$ & $\begin{array}{c}- \\
335.56\end{array}$ & $\begin{array}{c}- \\
338.38\end{array}$ & 2.82 & 0.84 & & & & & & \\
\hline
\end{tabular}


VI. RESULT AND DISCUSSION

\section{A. Evaluating PTDF and LODF}

The process began by defining an operating point (also called the Base Case: BC) of the network. Subsequently, the DC MW flow and the linear sensitivity factors from this operating point $(\mathrm{BC})$ are evaluated and stored. Table 1 gives the DC line flows for all 77 transmission line at the defined base case. The PTDF of 40 buses with respect to the slack bus (bus 27) while LODF of the 77 transmission lines can be calculated at this operating condition. The size of the resulting PTDF and LODF matrices cannot be reflected in this publication being 17 by 77 and 77 by 77 respectively. However, evaluating PTDF and LODF can be demonstrated. To this end, as table 2 shows, buses 2 and line 8 were chosen to demonstrate how PTDF and LODF of the 77 transmission lines are calculated. Using equation 1 , restated as

$$
P T D F_{i, k, l}=\frac{\Delta f_{l}}{\Delta P}=\frac{\widehat{f}_{l}-f_{l}^{0}}{\Delta P_{i \text { to } k}}
$$

$f_{l}^{0} \& \widehat{f}_{l}:$ Pre and Post generator outage MW flow on line $l(l=1,2, \ldots 77)$

$\Delta f_{l}=\widehat{f}_{l}-f_{l}^{0}:$ Change in MW flow of the monitored line when power is transferred

$\Delta P_{i \text { to } k}$ : Power transferred from Outage bus $i=2$ to the Reference bus $k=27$

Considering generator at bus 2 , its pre-outage output is 600MW, the post outage output is 0MW. $\Delta P_{2 \text { to } 27}=0-600=-600 \mathrm{MW}$. This is the power which the reference bus 27 now delivers at postoutage. If we were to monitor line $l=4$, then from table 1 , the pre-outage flow

$f_{4}^{0}=437.9 \mathrm{MW}$, post-outage flow $\widehat{f}_{4}=400.3 \mathrm{MW}$

$$
\begin{gathered}
P T D F_{2,27,4}=\frac{\widehat{f}_{4}-f_{4}^{0}}{\Delta P_{2 \text { to } 27}}=\frac{400.3-437.9}{-600} \\
=0.062667
\end{gathered}
$$

Similarly,

$$
L O D F_{l, t}=\frac{\Delta f_{l}}{f_{t}^{0}}=\frac{\widehat{f}_{l}-f_{l}^{0}}{f_{t}^{0}}
$$

$f_{l}^{0} \& \widehat{f}_{l}$ : Pre and Post outage MW flow on line $l(l=$ $1,2, \ldots 77)$

$\Delta f_{l}=\widehat{f}_{l}-f_{l}^{0}$ : Change in MW flow on monitored line $l$

$f_{t}^{0}=$ original flow on outage (open) line $t$

From Table 1 , consider line $t=8$, the pre-outage MW flow is $-91.94 \mathrm{MW}$ ( but actually flowing from bus 5 to bus 3 ). If we monitor line $l=4$, its preoutage flow $f_{4}^{0}=437.9 \mathrm{MW}$, and following the outage of line $t=8$, the post outage flow on line $l=$ $4 ; \widehat{f}_{4}=432.8 M W$. Then,

$$
L O D F_{4,8}=\frac{\widehat{f}_{4}-f_{4}^{0}}{f_{8}^{0}}=\frac{432.8-437.9}{-91.94}=0.005547
$$

The complete PTDF and LODF for the 77 lines with respect to bus 2 and line 8 is given in table 2 .

\section{B. Predicting Post Contingency MW Flow from Sensitivity Factors}

When contingency occurs in terms of line outage or generator/load outage, generator output/load variation then the evaluated and stored base case MW line flows and the sensitivity factors may be used to predict the line MW flow without resorting to the tedious load flow or state estimation techniques. Using PTDF, the MW line flow following power variation at any bus compensated by the reference bus $\mathrm{k}$ is

$$
\widehat{f}_{l}=P T D F_{i, k, l} * \Delta P_{i \text { to } k}+f_{l}^{0}
$$

While the MW line flow following line outage using LODF is

$$
\widehat{f}_{l}=L O D F_{l, t} * f_{t}^{0}+f_{l}^{0}
$$

Consider the following contingency conditions in table 4. Additionally, table 3 reports the PTDF with respect to buses 20 and 25 as well as the LODF with respect to lines 10,15 and 49 . 
International Journal of Trend in Scientific Research and Development (IJTSRD) ISSN: 2456-6470

Table 2: Calculating PTDF and LODF for Bus 2 and Line 8

\begin{tabular}{|c|c|c|c|c|c|c|c|c|c|c|c|}
\hline $\begin{array}{l}\text { Li } \\
\text { ne } \\
\text { Co } \\
\text { de }\end{array}$ & $\begin{array}{l}\text { Base } \\
\text { Case } \\
\text { flow }\end{array}$ & $\begin{array}{l}\text { Gen2 } \\
\text { Outage } \\
\text { Flow }\end{array}$ & $\begin{array}{l}\text { PT } \\
\text { DF }\end{array}$ & $\begin{array}{c}\text { L8 } \\
\text { Outage } \\
\text { Flow }\end{array}$ & $\begin{array}{l}\mathrm{LO} \\
\mathrm{DF}\end{array}$ & $\begin{array}{l}\text { Line } \\
\text { Code }\end{array}$ & $\begin{array}{l}\text { Base } \\
\text { Case } \\
\text { flow }\end{array}$ & $\begin{array}{l}\text { Gen2 } \\
\text { Outage } \\
\text { Flow }\end{array}$ & $\begin{array}{l}\text { PT } \\
\text { DF }\end{array}$ & $\begin{array}{c}\text { L8 } \\
\text { Outage } \\
\text { Flow }\end{array}$ & $\begin{array}{l}\text { LO } \\
\text { DF }\end{array}$ \\
\hline L1 & -150.0 & -150.0 & 0 & -150.0 & 0 & $\mathrm{~L} 40$ & 147.0 & 147.0 & 0 & 147.0 & 0 \\
\hline L2 & 225.0 & -75.0 & 0.5 & 225.0 & 0 & L41 & 197.0 & 197.0 & 0 & 197.0 & 0 \\
\hline L3 & 225.0 & -75.0 & 0.5 & 225.0 & 0 & $\mathrm{~L} 42$ & 197.0 & 197.0 & 0 & 197.0 & 0 \\
\hline L4 & 437.9 & 400.3 & $\begin{array}{c}0.0 \\
63\end{array}$ & 432.8 & $\begin{array}{c}0.0 \\
56\end{array}$ & L43 & 57.2 & 78.0 & $\begin{array}{c}- \\
0.0 \\
35\end{array}$ & 60.0 & $\begin{array}{c}- \\
0.0 \\
31\end{array}$ \\
\hline L5 & 437.9 & 400.3 & $\begin{array}{c}0.0 \\
63 \\
\end{array}$ & 432.8 & $\begin{array}{c}0.0 \\
56 \\
\end{array}$ & L44 & -320.0 & -320.0 & 0 & -320.0 & 0 \\
\hline L6 & -91.9 & -266.9 & $\begin{array}{l}0.2 \\
92\end{array}$ & -132.8 & $\frac{0.4}{44}$ & L45 & 504.1 & 558.5 & $\begin{array}{c}- \\
0.0 \\
91 \\
\end{array}$ & 511.6 & $\begin{array}{c}- \\
0.0 \\
81 \\
\end{array}$ \\
\hline L7 & -91.9 & -266.9 & $\begin{array}{l}0.2 \\
92 \\
\end{array}$ & -132.8 & $\begin{array}{l}0.4 \\
44 \\
\end{array}$ & L46 & 371.6 & 371.6 & 0 & 371.6 & 0 \\
\hline L8 & -91.9 & -266.9 & $\begin{array}{l}0.2 \\
92\end{array}$ & 0.0 & -1 & L47 & 300.0 & 300.0 & 0 & 300.0 & 0 \\
\hline L9 & 250.0 & 250 & 0 & 250.0 & 0 & L48 & -282.0 & -155.4 & $\begin{array}{c}- \\
0.2 \\
11\end{array}$ & -280.5 & $\begin{array}{c}- \\
0.0 \\
16 \\
\end{array}$ \\
\hline $\begin{array}{c}\mathrm{L} 1 \\
0\end{array}$ & 250.0 & 250.0 & 0 & 250.0 & $\begin{array}{c}1 \\
0 \\
\text { end }\end{array}$ & L49 & -282.0 & -155.4 & $\begin{array}{c}- \\
0.2 \\
11\end{array}$ & -280.5 & $\begin{array}{c}- \\
0.0 \\
16 \\
\end{array}$ \\
\hline $\begin{array}{c}\mathrm{L} 1 \\
1 \\
\end{array}$ & 175.0 & 175.0 & 0 & 175.0 & e e & $\mathrm{L} 50$ & n-176.1 & -476.1 & 0.5 & -176.1 & 0 \\
\hline $\begin{array}{c}\mathrm{L} 1 \\
2\end{array}$ & 175.0 & 175.0 & 0 & 175.0 & $\mathrm{e}_{0} \mathrm{C}$ & L51 & -176.1 & -476.1 & 0.5 & -176.1 & 0 \\
\hline $\begin{array}{c}\mathrm{L} 1 \\
3 \\
\end{array}$ & 275.4 & 237.8 & $\begin{array}{r}0.0 \\
63 \\
\end{array}$ & 270.3 & $\begin{array}{l}0.0 \\
56 \\
\end{array}$ & L52 & $7-235.0$ & -235.0 & 0 & -235.0 & 0 \\
\hline $\begin{array}{c}\text { L1 } \\
4 \\
\end{array}$ & 275.4 & 237.8 & $\begin{array}{l}0.0 \\
63 \\
\end{array}$ & 270.3 & $\begin{array}{l}0.0 \\
56 \\
\end{array}$ & $\mathrm{~L} 53$ & -235.0 & -235.0 & 0 & -235.0 & 0 \\
\hline $\begin{array}{c}\mathrm{L} 1 \\
5\end{array}$ & -174.9 & -234.2 & $\begin{array}{c}0.0 \\
99\end{array}$ & -172.5 & $\begin{array}{l}- \\
0.0 \\
27 \\
\end{array}$ & L54 & & 2.0 & $\begin{array}{l}0.1 \\
62\end{array}$ & 96.0 & $\begin{array}{c}- \\
0.0 \\
09 \\
\end{array}$ \\
\hline $\begin{array}{c}\mathrm{L} 1 \\
6\end{array}$ & -174.9 & -234.2 & $\begin{array}{c}0.0 \\
99\end{array}$ & -172.5 & $\begin{array}{l}- \\
0.0 \\
27 \\
\end{array}$ & L55 & 227.5 & 227.5 & 0 & 227.5 & 0 \\
\hline $\begin{array}{c}\mathrm{L} 1 \\
7\end{array}$ & -174.9 & -234.2 & $\begin{array}{c}0.0 \\
99\end{array}$ & -172.5 & $\begin{array}{c}- \\
0.0 \\
27 \\
\end{array}$ & L56 & 227.5 & 227.5 & 0 & 227.5 & 0 \\
\hline $\begin{array}{c}\mathrm{L} 1 \\
8\end{array}$ & 45.1 & -112.4 & $\begin{array}{c}0.2 \\
63\end{array}$ & 46.4 & $\begin{array}{c}- \\
0.0 \\
14 \\
\end{array}$ & L57 & 69.6 & 69.6 & 0 & 69.6 & 0 \\
\hline $\begin{array}{c}\text { L1 } \\
9\end{array}$ & 2.9 & -186.5 & $\begin{array}{c}0.3 \\
16\end{array}$ & 4.5 & $\begin{array}{c}- \\
0.0 \\
17 \\
\end{array}$ & L58 & 201.7 & 210.5 & $\begin{array}{c}- \\
0.0 \\
15 \\
\end{array}$ & 202.9 & $\begin{array}{c}- \\
0.0 \\
13 \\
\end{array}$ \\
\hline $\begin{array}{c}\mathrm{L} 2 \\
0\end{array}$ & 350.0 & 350.0 & 0 & 350.0 & 0 & L59 & 123.7 & 129.8 & $\begin{array}{c}- \\
0.0 \\
1 \\
\end{array}$ & 124.6 & $\begin{array}{c}- \\
0.0 \\
09 \\
\end{array}$ \\
\hline
\end{tabular}


International Journal of Trend in Scientific Research and Development (IJTSRD) ISSN: 2456-6470

\begin{tabular}{|c|c|c|c|c|c|c|c|c|c|c|c|}
\hline $\begin{array}{c}\mathrm{L} 2 \\
1\end{array}$ & 66.9 & 41.9 & $\begin{array}{l}0.0 \\
42\end{array}$ & 63.5 & $\begin{array}{l}0.0 \\
37\end{array}$ & L60 & 123.7 & 129.8 & $\begin{array}{c}- \\
0.0 \\
1 \\
\end{array}$ & 124.6 & $\begin{array}{c}- \\
0.0 \\
09 \\
\end{array}$ \\
\hline $\begin{array}{c}\mathrm{L} 2 \\
2\end{array}$ & 66.9 & 41.9 & $\begin{array}{l}0.0 \\
42\end{array}$ & 63.5 & $\begin{array}{c}0.0 \\
37\end{array}$ & L61 & -0.4 & 10.0 & $\begin{array}{c}- \\
0.0 \\
17 \\
\end{array}$ & 1.0 & $\begin{array}{c}- \\
0.0 \\
16 \\
\end{array}$ \\
\hline $\begin{array}{c}\mathrm{L} 2 \\
3\end{array}$ & 66.9 & 41.9 & $\begin{array}{c}0.0 \\
42\end{array}$ & 63.5 & $\begin{array}{c}0.0 \\
37\end{array}$ & L62 & -0.4 & 10.0 & $\begin{array}{c}- \\
0.0 \\
17 \\
\end{array}$ & 1.0 & $\begin{array}{c}- \\
0.0 \\
16 \\
\end{array}$ \\
\hline $\begin{array}{c}\mathrm{L} 2 \\
4 \\
\end{array}$ & 269.4 & 256.0 & $\begin{array}{l}0.0 \\
22 \\
\end{array}$ & 267.6 & $\begin{array}{c}0.0 \\
2 \\
\end{array}$ & L63 & 49.0 & 49.0 & 0 & 49.0 & 0 \\
\hline $\begin{array}{c}\mathrm{L} 2 \\
5 \\
\end{array}$ & -159.3 & -190.2 & $\begin{array}{c}0.0 \\
52 \\
\end{array}$ & -163.5 & $\begin{array}{c}0.0 \\
46 \\
\end{array}$ & L64 & 49.0 & 49.0 & 0 & 49.0 & 0 \\
\hline $\begin{array}{c}\mathrm{L} 2 \\
6 \\
\end{array}$ & -159.3 & -190.2 & $\begin{array}{c}0.0 \\
52 \\
\end{array}$ & -163.5 & $\begin{array}{c}0.0 \\
46 \\
\end{array}$ & L65 & -282.1 & -292.5 & $\begin{array}{c}0.0 \\
17 \\
\end{array}$ & -283.5 & $\begin{array}{l}0.0 \\
16 \\
\end{array}$ \\
\hline $\begin{array}{c}\mathrm{L} 2 \\
7 \\
\end{array}$ & -160.0 & -160.0 & 0 & & 0 & L66 & -282 & 292.5 & $\begin{array}{c}0.0 \\
17 \\
\end{array}$ & -283.5 & $\begin{array}{l}0.0 \\
16 \\
\end{array}$ \\
\hline $\begin{array}{c}\mathrm{L} 2 \\
8\end{array}$ & 142.7 & 50.5 & & & $\begin{array}{c}0.0 \\
08 \\
\end{array}$ & L67 & $\begin{array}{r}-127.3 \\
\end{array}$ & & $\begin{array}{l}0.1 \\
54\end{array}$ & -126.5 & $\begin{array}{c} \\
0.0 \\
08 \\
\end{array}$ \\
\hline $\begin{array}{c}\mathrm{L} 2 \\
9 \\
\end{array}$ & 50.6 & 64.0 & $\begin{array}{c}- \\
0.0 \\
22 \\
\end{array}$ & 52.5 & $\begin{array}{c}0.0 \\
2 \\
\end{array}$ & L68 & -8.8 & $\rightarrow$ & $\begin{array}{l}0.1 \\
62 \\
\end{array}$ & -8.0 & $\begin{array}{c}- \\
0.0 \\
09 \\
\end{array}$ \\
\hline $\begin{array}{c}\text { L3 } \\
0 \\
\end{array}$ & 200.0 & 200.0 & 0 & 200.0 & 0 & L69 & 200.0 & 200.0 & 0 & 200.0 & 0 \\
\hline $\begin{array}{c}\mathrm{L} 3 \\
1 \\
\end{array}$ & -380.6 & -394.0 & $\begin{array}{l}0.0 \\
22 \\
\end{array}$ & -382.5 & $\begin{array}{c}0.0 \\
2 \\
\end{array}$ & $\mathrm{L70}$ & -439.2 & -476.7 & $\begin{array}{l}0.0 \\
63 \\
\end{array}$ & -444.3 & $\begin{array}{c}0.0 \\
56 \\
\end{array}$ \\
\hline $\begin{array}{c}\text { L3 } \\
2 \\
\end{array}$ & -147.0 & -147.0 & 0 & -147.0 & $e^{0}$ & L71 & -439.2 & -476.7 & $\begin{array}{l}0.0 \\
63 \\
\end{array}$ & -444.3 & $\begin{array}{l}0.0 \\
56 \\
\end{array}$ \\
\hline $\begin{array}{c}\mathrm{L} 3 \\
3\end{array}$ & -323.2 & -323.2 & 0 & & & L72 & 189.9 & & $\begin{array}{c}- \\
0.0 \\
11 \\
\end{array}$ & 190.8 & $\begin{array}{c} \\
0.0 \\
1 \\
\end{array}$ \\
\hline $\begin{array}{c}\mathrm{L} 3 \\
4\end{array}$ & -323.2 & -323.2 & 0 & & & L73 & 189.9 & 196.6 & $\begin{array}{c}- \\
0.0 \\
11 \\
\end{array}$ & 190.8 & $\begin{array}{c}- \\
0.0 \\
1 \\
\end{array}$ \\
\hline $\begin{array}{c}\mathrm{L} 3 \\
5\end{array}$ & -323.2 & -323.2 & 0 & & & L74 & 146.7 & 155.5 & $\begin{array}{c}- \\
0.0 \\
15 \\
\end{array}$ & 147.9 & $\begin{array}{c}- \\
0.0 \\
13\end{array}$ \\
\hline $\begin{array}{c}\text { L3 } \\
6\end{array}$ & 118.8 & 143.8 & $\begin{array}{c}- \\
0.0 \\
42 \\
\end{array}$ & 122.2 & $\begin{array}{c}- \\
0.0 \\
37 \\
\end{array}$ & L75 & -155.0 & -155.0 & 0 & -155.0 & 0 \\
\hline $\begin{array}{c}\mathrm{L} 3 \\
7\end{array}$ & 118.8 & 143.8 & $\begin{array}{c}- \\
0.0 \\
42 \\
\end{array}$ & 122.2 & $\begin{array}{c}- \\
0.0 \\
37 \\
\end{array}$ & L76 & -155.0 & -155.0 & 0 & -155.0 & 0 \\
\hline $\begin{array}{l}\text { L3 } \\
8\end{array}$ & 118.8 & 143.8 & $\begin{array}{c}- \\
0.0 \\
42 \\
\end{array}$ & 122.2 & $\begin{array}{c}- \\
0.0 \\
37 \\
\end{array}$ & L77 & -82.0 & -82.0 & 0 & -82.0 & 0 \\
\hline $\begin{array}{c}\text { L3 } \\
9\end{array}$ & -338.4 & -338.4 & 0 & -338.4 & 0 & & & & & & \\
\hline
\end{tabular}


International Journal of Trend in Scientific Research and Development (IJTSRD) ISSN: 2456-6470 Table 3: Selected PTDF (Buses 20 \& 25) and LODF (Line 10.15 \& 49)

\begin{tabular}{|c|c|c|c|c|c|c|c|c|c|c|c|}
\hline $\mathbf{L C}$ & Bus 20 & Bus 25 & L10 & L15 & L49 & $\mathrm{LC}$ & Bus 20 & Bus 25 & L10 & L15 & L49 \\
\hline $\mathbf{L} \mathbf{1}$ & 0 & 0 & 0 & 0 & 0 & L40 & 0 & 0 & 0 & 0 & 0 \\
\hline $\mathbf{L} 2$ & 0 & 0 & 0 & 0 & 0 & L41 & 0 & 0 & 0 & 0 & 0 \\
\hline $\mathbf{L 3}$ & 0 & 0 & 0 & 0 & 0 & L42 & 0 & 0 & 0 & 0 & 0 \\
\hline L4 & -0.0204 & 0.0071 & 0 & 0.0565 & 0.0304 & L43 & 0.0113 & -0.0039 & 0 & -0.0313 & -0.0168 \\
\hline L5 & -0.0204 & 0.0071 & 0 & 0.0565 & 0.0304 & L44 & 0 & 0 & 0 & 0 & 0 \\
\hline L6 & 0.0136 & -0.0047 & 0 & -0.0377 & -0.0202 & L45 & 0.0295 & -0.0102 & 0 & -0.0817 & -0.0439 \\
\hline L7 & 0.0136 & -0.0047 & 0 & -0.0377 & -0.0202 & L46 & -0.0803 & 0 & 0 & 0 & 0 \\
\hline L8 & 0.0136 & -0.0047 & 0 & -0.0377 & -0.0202 & L47 & 0 & 0 & 0 & 0 & 0 \\
\hline L9 & 0 & 0 & 1 & 0 & 0 & L48 & -0.3288 & -0.0595 & 0 & 0.1647 & 0.4898 \\
\hline L10 & 0 & 0 & -1 & 0 & 0 & L49 & -0.3288 & -0.0595 & 0 & 0.1647 & -1 \\
\hline L11 & 0 & 0 & 0 & 0 & 0 & L50 & 0.5 & 0.5 & 0 & 0 & 0 \\
\hline L12 & 0 & 0 & 0 & 0 & $\begin{array}{l}\text { er } \\
-\end{array}$ & L51 & 0.5 & 0.5 & 0 & 0 & 0 \\
\hline L13 & -0.0204 & 0.0071 & 0 & 0.0565 & 0.0304 & $\mathbf{L 5 2}$ & 0 & 0 & 0 & 0 & 0 \\
\hline L14 & -0.0204 & 0.0071 & 0 & 0.0565 & 0.0304 & L53 & 0 & 0 & 0 & 0 & 0 \\
\hline L15 & -0.1006 & 0.0349 & 0 & -1 & 0.1498 & L54 & 0.0959 & 0.5726 & 0 & 0.0923 & -0.1429 \\
\hline L16 & -0.1006 & 0.0349 & 0 & 0.2788 & 0.1498 & $\mathbf{L 5 5}$ & 0 & 0 & 0 & 0 & 0 \\
\hline L17 & -0.1006 & 0.0349 & 0 & 0.2788 & 0.1498 & L56 & 0 & 0 & 0 & 0 & 0 \\
\hline L18 & 0.1555 & 0.081 & 0 & 0.1496 & -0.2317 & L57 & -0.0803 & 0 & 0 & 0 & 0 \\
\hline L19 & 0.187 & -0.1999 & 0 & 0.1799 & -0.2786 & L58 & 0.0048 & -0.0016 & 0 & -0.0132 & -0.0071 \\
\hline L20 & 0 & 0 & 0 & 0 & 0 & L59 & 0.0033 & -0.0011 & 0 & -0.0091 & -0.0049 \\
\hline L21 & -0.0136 & 0.0047 & 0 & 0.0377 & 0.0202 & L60 & 0.0033 & -0.0011 & 0 & -0.0091 & -0.0049 \\
\hline L22 & -0.0136 & 0.0047 & 0 & 0.0377 & 0.0202 & L61 & 0.0056 & -0.002 & 0 & -0.0157 & -0.0084 \\
\hline L23 & -0.0136 & 0.0047 & 0 & 0.0377 & 0.0202 & L62 & 0.0056 & -0.002 & 0 & -0.0157 & -0.0084 \\
\hline L24 & -0.0072 & 0.0025 & 0 & 0.0201 & 0.0108 & L63 & 0 & 0 & 0 & 0 & 0 \\
\hline L25 & -0.0168 & 0.0058 & 0 & 0.0465 & 0.025 & L64 & 0 & 0 & 0 & 0 & 0 \\
\hline L26 & -0.0168 & 0.0058 & 0 & 0.0465 & 0.025 & L65 & -0.0056 & 0.002 & 0 & 0.0157 & 0.0084 \\
\hline
\end{tabular}


International Journal of Trend in Scientific Research and Development (IJTSRD) ISSN: 2456-6470

\begin{tabular}{|c|c|c|c|c|c|c|c|c|c|c|c|}
\hline L27 & 0 & 0 & 0 & 0 & 0 & L66 & -0.0056 & 0.002 & 0 & 0.0157 & 0.0084 \\
\hline L28 & 0.0911 & 0.2274 & 0 & 0.0876 & -0.1357 & L67 & 0.0911 & 0.2274 & 0 & 0.0876 & -0.1357 \\
\hline L29 & 0.0072 & -0.0025 & 0 & -0.0201 & -0.0108 & L68 & 0.0959 & -0.4274 & 0 & 0.0923 & -0.1429 \\
\hline L30 & 0 & 0 & 0 & 0 & 0 & L69 & 0 & 0 & 0 & 0 & 0 \\
\hline L31 & -0.0072 & 0.0025 & 0 & 0.0201 & 0.0108 & L70 & -0.0204 & 0.0071 & 0 & 0.0565 & 0.0304 \\
\hline L32 & 0 & 0 & 0 & 0 & 0 & L71 & -0.0204 & 0.0071 & 0 & 0.0565 & 0.0304 \\
\hline L33 & -0.3066 & 0 & 0 & 0 & 0 & L72 & 0.0036 & -0.0013 & 0 & -0.01 & -0.0054 \\
\hline L34 & -0.3066 & 0 & 0 & 0 & 0 & L73 & 0.0036 & -0.0013 & 0 & -0.01 & -0.0054 \\
\hline L35 & -0.3066 & 0 & 0 & 0 & 0 & L74 & 0.0048 & -0.0016 & 0 & -0.0132 & -0.0071 \\
\hline L36 & 0.0136 & -0.0047 & 0 & -0.0377 & -0.0202 & L75 & $7 /$ & C & 0 & 0 & 0 \\
\hline L37 & 0.0136 & -0.0047 & 0 & -0.0377 & -0.0202 & L76 & 0 & 0 & 0 & 0 & 0 \\
\hline L38 & 0.0136 & -0.0047 & 0 & -0.0377 & -0.0202 & L77 & 0 & 0 & 0 & 0 & 0 \\
\hline L39 & -0.0803 & 0 & 0 & 0 & 0 & & & & & & \\
\hline
\end{tabular}

Table 4: Contingency definition for Post Contingency Flow Prediction

\begin{tabular}{|c|c|c|c|c|c|}
\hline \multicolumn{2}{|c|}{ Contingency } & \multicolumn{2}{c|}{ MW Output } & Exchanged & \multirow{2}{*}{$\begin{array}{c}\text { Slack bus } \\
\text { action }\end{array}$} \\
\hline Definition & Location & Pre-contingency & Post-Contingency & Power & supply more \\
\hline 50\% output Reduction & Bus 2 & 600 & 300 & -300 & supply less \\
\hline 100MW output Increment & Bus 20 & 900 & 1000 & 100 & supply \\
\hline 100MW output Reduction & Bus 25 & 304 & -100 & supply more \\
\hline 140 MW Load Reduction & Bus 1 & 150 & 10 & $140 *$ & supply less \\
\hline
\end{tabular}

*The change with respect to load is positive, unlike generator.

From the base case and proceed with these mutually exclusive contingencies. Let's assume that the load center at bus 1 reduced its demand from the base case of $150 \mathrm{MW}$ to $10 \mathrm{MW}$ or that the outputs at generator buses 2, 20 and 25 were varied as stated on the table; the idea is to predict the MW flow on any or all of the transmission lines without resort to state estimation or load flow. In order to accomplish this, we need information of the flow at any operation and the corresponding linear sensitivity factors.

If we decide to predict the flows on line 54, when any of the contingencies of table 4 occur, then we would need the

Pre-outage flow on the monitored line $f_{l}^{0}=95.16 M W$

The power exchanged between the contingency bus and the reference bus: ( $5^{\text {th }}$ column) of table 4

The corresponding sensitivity factors, (PTDF) of line 54 with respect to the contingency bus 1 , bus 2 , bus 20 and bus 25 which are $0.1619,0.162,0.0959$ and 0.5726 respectively.

$$
\text { Bus } \begin{aligned}
1: \widehat{f_{54}} & =P T D F_{1,27,54} * \Delta P_{1 \text { to } 27}+f_{54}^{0} \\
& =0.1619 *(150-10)+95.16 \\
& =117.826 M W
\end{aligned}
$$

Bus 2: $\widehat{f_{54}}=P T D F_{2,27,54} * \Delta P_{2 \text { to } 27}+f_{54}^{0}$

$$
\begin{aligned}
& =0.162 *(300-600)+95.16 \\
& =46.56 \mathrm{MW}
\end{aligned}
$$


International Journal of Trend in Scientific Research and Development (IJTSRD) ISSN: 2456-6470

Bus 20: $\widehat{f_{54}}=P T D F_{20,27,54} * \Delta P_{20 \text { to } 27}+f_{54}^{0}$

$$
=37.90 \mathrm{MW}
$$

$$
\begin{aligned}
& =0.0959 *(1000-900)+95.16 \\
& =104.75 M W
\end{aligned}
$$

Notice that the values of flow estimated from PTDF match the DC load flow output reported in table 6.

\begin{tabular}{|c|c|c|c|c|c|c|c|c|c|c|c|}
\hline \multirow{2}{*}{$\begin{array}{c}\text { Lin } \\
\text { e } \\
\text { Cod } \\
\text { e }\end{array}$} & \multirow{2}{*}{$\begin{array}{l}\text { BC } \\
\text { MW } \\
\text { flow }\end{array}$} & \multicolumn{4}{|c|}{ Predicted MW Flow } & \multirow{2}{*}{$\begin{array}{c}\text { Lin } \\
\mathrm{e} \\
\text { Cod } \\
\mathrm{e}\end{array}$} & \multirow{2}{*}{$\begin{array}{c}\text { BC } \\
\mathrm{M} \\
\mathrm{W} \\
\text { flo } \\
\mathrm{W} \\
\end{array}$} & \multicolumn{4}{|c|}{ Predicted MW Flow } \\
\hline & & $\begin{array}{c}140 \mathrm{M} \\
\text { W load } \\
\text { Red at } \\
\text { bus } 1\end{array}$ & $\begin{array}{c}50 \% \text { Gen } \\
\text { Red at } \\
\text { bus } 2\end{array}$ & $\begin{array}{l}100 \mathrm{mw} \\
\text { Inc at } \\
\text { bus } 20\end{array}$ & $\begin{array}{l}100 \mathrm{mw} \\
\text { Red at } \\
\text { bus } 25\end{array}$ & & & $\begin{array}{l}140 \mathrm{MW} \\
\text { load Red } \\
\text { at bus } 1\end{array}$ & $\begin{array}{c}50 \% \text { Gen } \\
\text { Red at } \\
\text { bus } 2\end{array}$ & $\begin{array}{l}100 \mathrm{mw} \\
\text { Inc at } \\
\text { bus } 20\end{array}$ & $\begin{array}{l}100 \mathrm{mw} \\
\text { Red at } \\
\text { bus } 25\end{array}$ \\
\hline L1 & $\begin{array}{c}- \\
150 . \\
00\end{array}$ & -10.00 & 150.0 & & 150.00 & L40 & $\begin{array}{r}147 \\
.00 \\
\end{array}$ & 147.00 & -147.00 & -147.00 & -147.00 \\
\hline $\mathrm{L} 2$ & $\begin{array}{c}225 . \\
00\end{array}$ & 295.00 & -75.00 & -225.00 & 225.00 & $\mathrm{~L} 41$ & $\begin{array}{l}197 \\
.00\end{array}$ & 197.00 & 7.00 & -197.00 & -197.00 \\
\hline L3 & $\begin{array}{c}225 . \\
00\end{array}$ & 295.00 & 75.0 & -225.00 & 225.00 & L42 & $\begin{array}{l}197 \\
.00 \\
\end{array}$ & 197.00 & -197.00 & -197.00 & -197.00 \\
\hline L4 & $\begin{array}{c}437 . \\
90 \\
\end{array}$ & 446.67 & -419.13 & -435.87 & $\begin{array}{c}- \\
437.20 \\
\end{array}$ & $\mathrm{~L} 43$ & $\begin{array}{l}57 . \\
20 \\
\end{array}$ & 52.34 & -67.59 & -58.31 & -57.57 \\
\hline L5 & $\begin{array}{c}437 . \\
90\end{array}$ & 446.67 & -419.13 & -435.87 & 437.20 & $\mathrm{~L} 44$ & $\begin{array}{c}320 \\
000 \\
\end{array}$ & -320.00 & 320.00 & 320.00 & 320.00 \\
\hline L6 & $\begin{array}{c}- \\
91.9 \\
0 \\
\end{array}$ & -51.12 & 179.42 & 90.58 & 91.47 & $\mathrm{~L} 45$ & $\begin{array}{l}504 \\
.10\end{array}$ & 491.42 & -531.29 & -507.06 & -505.13 \\
\hline $\mathrm{L} 7$ & $\begin{array}{c}- \\
91.9 \\
0 \\
\end{array}$ & -51.12 & 179.42 & 90.58 & 91.47 & L46 & $\begin{array}{l}371 \\
.60 \\
\end{array}$ & 371.60 & -371.62 & -363.59 & -371.62 \\
\hline L8 & $\begin{array}{c}- \\
91.9 \\
0 \\
\end{array}$ & -51.12 & 179.42 & & 91.47 & L47 & $\begin{array}{r}300 \\
.00\end{array}$ & 300.00 & -300.00 & -300.00 & -300.00 \\
\hline L9 & $\begin{array}{c}250 . \\
00\end{array}$ & 250.00 & -250.00 & -250.00 & 250.00 & L48 & $\begin{array}{l}282 \\
.00 \\
\end{array}$ & -311.53 & 218.71 & 314.86 & 276.03 \\
\hline L10 & $\begin{array}{c}250 . \\
00\end{array}$ & 250.00 & -250.00 & -250.00 & $\begin{array}{c}- \\
250.00\end{array}$ & L49 & $\begin{array}{c}- \\
282 \\
.00 \\
\end{array}$ & -311.53 & 218.71 & 314.86 & 276.03 \\
\hline L11 & $\begin{array}{c}175 . \\
00\end{array}$ & 175.00 & -175.00 & -175.00 & $\begin{array}{c}- \\
175.00\end{array}$ & L50 & $\begin{array}{c}- \\
176 \\
.10 \\
\end{array}$ & -106.10 & 326.06 & 126.06 & 226.06 \\
\hline L12 & $\begin{array}{c}175 . \\
00\end{array}$ & 175.00 & -175.00 & -175.00 & $\begin{array}{c}- \\
175.00\end{array}$ & L51 & $\begin{array}{c}- \\
176 \\
.10 \\
\end{array}$ & -106.10 & 326.06 & 126.06 & 226.06 \\
\hline L13 & $\begin{array}{c}275 . \\
40\end{array}$ & 284.17 & -256.63 & -273.37 & $\begin{array}{c}- \\
274.70\end{array}$ & L52 & $\begin{array}{c}- \\
235 \\
.00 \\
\end{array}$ & -235.00 & 235.00 & 235.00 & 235.00 \\
\hline
\end{tabular}

Bus 25: $\begin{aligned} \widehat{f_{54}} & =P T D F_{25,27,54} * \Delta P_{25} \text { to } 27+f_{54}^{0} \\ & =0.5726 *(204-304)+95.16\end{aligned}$

Table 5: Predicted MW Flow at variable Generator Output 
International Journal of Trend in Scientific Research and Development (IJTSRD) ISSN: 2456-6470

\begin{tabular}{|c|c|c|c|c|c|c|c|c|c|c|c|}
\hline L14 & $\begin{array}{c}275 . \\
40\end{array}$ & 284.17 & -256.63 & -273.37 & $\begin{array}{c}- \\
274.70\end{array}$ & L53 & $\begin{array}{c}- \\
235 \\
.00 \\
\end{array}$ & -235.00 & 235.00 & 235.00 & 235.00 \\
\hline L15 & $\begin{array}{c}- \\
174 . \\
90\end{array}$ & -161.06 & 204.58 & 184.97 & 178.40 & L54 & $\begin{array}{l}95 . \\
20\end{array}$ & 117.87 & -46.59 & -104.75 & -37.90 \\
\hline L16 & $\begin{array}{c}- \\
174 . \\
90\end{array}$ & -161.06 & 204.58 & 184.97 & 178.40 & L55 & $\begin{array}{r}227 \\
.50\end{array}$ & 227.50 & -227.50 & -227.50 & -227.50 \\
\hline L17 & $\begin{array}{c}- \\
174 . \\
90\end{array}$ & -161.06 & 204.58 & 184.97 & 178.40 & L56 & $\begin{array}{r}227 \\
.50\end{array}$ & 227.50 & -227.50 & -227.50 & -227.50 \\
\hline L18 & $\begin{array}{c}45.1 \\
0 \\
\end{array}$ & 81.81 & 33.69 & -60.61 & -36.96 & L57 & $\begin{array}{l}69 . \\
60 \\
\end{array}$ & 69.60 & -69.62 & -61.59 & -69.62 \\
\hline L19 & 2.90 & 47.06 & 91.85 & -21.56 & -22.85 & L58 & $\begin{array}{r}201 \\
.70 \\
\end{array}$ & 199.66 & -206.12 & -202.22 & -201.90 \\
\hline L20 & $\begin{array}{c}350 . \\
00\end{array}$ & 350.00 & -35 & 0 & 350.00 & L59 & $\begin{array}{l}123 \\
.70 \\
\end{array}$ & 122.3 & -126.72 & -124.05 & -123.83 \\
\hline L21 & $\begin{array}{c}66.9 \\
0 \\
\end{array}$ & 72.79 & & 50 & -66.47 & L60 & $\begin{array}{r}123 \\
.70 \\
\end{array}$ & & .72 & -124.05 & -123.83 \\
\hline L22 & $\begin{array}{c}66.9 \\
0\end{array}$ & 72.79 & & & -66.47 & L61 & $\begin{array}{c}- \\
0.4 \\
0\end{array}$ & -2 & & -0.15 & 0.21 \\
\hline L23 & $\begin{array}{c}66.9 \\
0\end{array}$ & 72.79 & -54.40 & -65.58 & $\begin{array}{l}-66.47 \\
\text { netho }\end{array}$ & L62 & $\begin{array}{l}.1 \% \\
0.4 \\
0\end{array}$ & -2.82 & & -0.15 & 0.21 \\
\hline L24 & $\begin{array}{c}269 . \\
40 \\
\end{array}$ & 272.50 & -262.69 & -268.66 & 269.13 & L63 & $\begin{array}{r}49 . \\
00\end{array}$ & 49.00 & -49.00 & -49.00 & -49.00 \\
\hline L25 & $\begin{array}{c}- \\
159 . \\
30\end{array}$ & -152.07 & 174.73 & 160.96 & $\begin{array}{c}\text { EVE } \\
159.86 \\
\mathrm{CN} \cdot 2\end{array}$ & $\begin{array}{l}\text { L64 } \\
6-6\end{array}$ & $\begin{array}{l}49 . \\
00\end{array}$ & 49.0 & -49.00 & -49.00 & -49.00 \\
\hline L26 & $\begin{array}{c}- \\
159 . \\
30\end{array}$ & -152.07 & 174.73 & 160 & 159.86 & L65 & $\begin{array}{c}- \\
282 \\
.10\end{array}$ & -279 & .28 & 282.65 & 282.29 \\
\hline L27 & $\begin{array}{c}- \\
160 . \\
00\end{array}$ & -160.00 & 160.00 & 160.00 & 160.00 & L66 & $\begin{array}{r}- \\
282 \\
.10 \\
\end{array}$ & -279.68 & 287.28 & 282.65 & 282.29 \\
\hline L28 & $\begin{array}{c}142 . \\
70\end{array}$ & 164.22 & -96.59 & -151.81 & 119.96 & L67 & $\begin{array}{l}- \\
127 \\
.30\end{array}$ & -105.78 & 173.41 & 118.19 & 150.04 \\
\hline L29 & $\begin{array}{c}50.6 \\
0\end{array}$ & 47.50 & -57.31 & -51.34 & -50.87 & L68 & $\begin{array}{c}- \\
8.8 \\
0\end{array}$ & 13.87 & 57.41 & -0.75 & -33.90 \\
\hline L30 & $\begin{array}{c}200 . \\
00 \\
\end{array}$ & 200.00 & -200.00 & -200.00 & $\begin{array}{c}- \\
200.00 \\
\end{array}$ & L69 & $\begin{array}{c}200 \\
.00 \\
\end{array}$ & 200.00 & -200.00 & -200.00 & -200.00 \\
\hline L31 & $\begin{array}{c}- \\
380 . \\
60 \\
\end{array}$ & -377.50 & 387.31 & 381.34 & 380.87 & L70 & $\begin{array}{c}- \\
439 \\
.20 \\
\end{array}$ & -430.44 & 457.93 & 441.19 & 439.86 \\
\hline L32 & $\begin{array}{c}- \\
147 . \\
00\end{array}$ & -147.00 & 147.00 & 147.00 & 147.00 & L71 & $\begin{array}{c}- \\
439 \\
.20\end{array}$ & -430.44 & 457.93 & 441.19 & 439.86 \\
\hline
\end{tabular}


International Journal of Trend in Scientific Research and Development (IJTSRD) ISSN: 2456-6470

\begin{tabular}{|c|c|c|c|c|c|c|c|c|c|c|c|}
\hline L33 & $\begin{array}{c}- \\
323 . \\
20\end{array}$ & -323.21 & 323.21 & 353.87 & 323.21 & L72 & $\begin{array}{r}189 \\
.90\end{array}$ & 188.35 & -193.20 & -190.23 & -190.00 \\
\hline L34 & $\begin{array}{c}- \\
323 . \\
20\end{array}$ & -323.21 & 323.21 & 353.87 & 323.21 & L73 & $\begin{array}{r}189 \\
.90\end{array}$ & 188.35 & -193.20 & -190.23 & -190.00 \\
\hline L35 & $\begin{array}{c}- \\
323 . \\
20\end{array}$ & -323.21 & 323.21 & 353.87 & 323.21 & L74 & $\begin{array}{c}146 \\
.70\end{array}$ & 144.66 & -151.12 & -147.22 & -146.90 \\
\hline L36 & $\begin{array}{c}118 . \\
80\end{array}$ & 112.92 & -131.31 & -120.13 & $\begin{array}{c}- \\
119.24\end{array}$ & L75 & $\begin{array}{c}- \\
155 \\
.00 \\
\end{array}$ & -155.00 & 155.00 & 155.00 & 155.00 \\
\hline L37 & $\begin{array}{c}118 . \\
80\end{array}$ & 112.92 & -131.31 & -120.13 & $\begin{array}{c}- \\
119.24\end{array}$ & L76 & $\begin{array}{c}- \\
155 \\
.00\end{array}$ & -155.00 & 155.00 & 155.00 & 155.00 \\
\hline L38 & $\begin{array}{c}118 . \\
80\end{array}$ & 112.92 & & & 119.24 & L77 & $\begin{array}{l}82 . \\
00\end{array}$ & & 82.00 & 82.00 & 82.00 \\
\hline L39 & $\begin{array}{c}- \\
338 . \\
40 \\
\end{array}$ & -338.38 & 338.38 & 346.41 & 338.38 & $\square$ & & & & & \\
\hline
\end{tabular}

Note: The negative sign on the flows simply mean that power flows in the opposite direction.

Table 6: Post Contingency Transmission Line Flow from DC load Flow Analysis

\begin{tabular}{|c|c|c|c|c|c|c|c|c|c|}
\hline $\mathrm{LC}$ & $\mathrm{BC}$ & Bus 2 & Bus 20 & Bus25 & LC & Bus 2 & Bus 20 & Bus25 & Bus 2 \\
\hline $\mathbf{L} 1$ & -150 & -150.00 & -150.00 & -150.00 & L40 & 147 & 147.00 & 147 & 147.00 \\
\hline $\mathbf{L 2}$ & 225 & 75.00 & 225.00 & 225.00 & L41 & 197 & 197.00 & 197.00 & 197.00 \\
\hline $\mathbf{L 3}$ & 225 & 75.00 & 225.00 & 225.00 & $\mathbf{L 4 2}$ & 197 & 197.00 & 197.00 & 197.00 \\
\hline L4 & 437.9 & 419.12 & 435.87 & 437.20 & L43 & 57.2 & 67 & 58.31 & 57.58 \\
\hline $\mathbf{L 5}$ & 437.9 & 419.12 & 435.87 & 437.20 & L44 & -320 & -320.00 & -320.00 & -320.00 \\
\hline L6 & -91.9 & -179.42 & -90.58 & -91.47 & L45 & 504.1 & 531.28 & 507.06 & 505.14 \\
\hline L7 & -91.9 & -179.42 & -90.58 & -91.47 & L46 & 371.6 & 371.62 & 363.59 & 371.62 \\
\hline L8 & -91.9 & -179.42 & -90.58 & -91.47 & L47 & 300 & 300.00 & 300.00 & 300.00 \\
\hline L9 & 250 & 250.00 & 250.00 & 250.00 & L48 & -282 & -218.70 & -314.86 & -276.03 \\
\hline L10 & 250 & 250.00 & 250.00 & 250.00 & L49 & -282 & -21 & -314.86 & -276.03 \\
\hline L11 & 175 & 175.00 & 175.00 & 175.00 & $\mathbf{L 5 0}$ & -176 & -326.06 & -126.06 & -226.06 \\
\hline L12 & 175 & 175.00 & 175.00 & 175.00 & L51 & -176 & -326.06 & -126.06 & -226.06 \\
\hline L13 & 275.4 & 256.62 & 273.37 & 274.70 & L52 & -235 & -235.00 & -235.00 & -235.00 \\
\hline L14 & 275.4 & 256.62 & 273.37 & 274.70 & L53 & -235 & -235.00 & -235.00 & -235.00 \\
\hline L15 & -175 & -204.57 & -184.97 & -178.41 & L54 & 95.2 & 46.59 & 104.75 & 37.90 \\
\hline L16 & -175 & -204.57 & -184.97 & -178.41 & L55 & 227.5 & 227.50 & 227.50 & 227.50 \\
\hline L17 & -175 & -204.57 & -184.97 & -178.41 & L56 & 227.5 & 227.50 & 227.50 & 227.50 \\
\hline
\end{tabular}


International Journal of Trend in Scientific Research and Development (IJTSRD) ISSN: 2456-6470

\begin{tabular}{|c|c|c|c|c|c|c|c|c|c|}
\hline L18 & 45.1 & -33.69 & 60.61 & 36.96 & L57 & 69.6 & 69.62 & 61.58 & 69.62 \\
\hline L19 & 2.9 & -91.84 & 21.56 & 22.85 & L58 & 201.7 & 206.12 & 202.22 & 201.91 \\
\hline L20 & 350 & 350.00 & 350.00 & 350.00 & L59 & 123.7 & 126.74 & 124.05 & 123.83 \\
\hline L21 & 66.9 & 54.42 & 65.58 & 66.47 & L60 & 123.7 & 126.74 & 124.05 & 123.83 \\
\hline L22 & 66.9 & 54.42 & 65.58 & 66.47 & L61 & -0.4 & 4.80 & 0.16 & -0.21 \\
\hline L23 & 66.9 & 54.42 & 65.58 & 66.47 & L62 & -0.4 & 4.80 & 0.16 & -0.21 \\
\hline L24 & 269.4 & 262.70 & 268.66 & 269.13 & L63 & 49 & 49.00 & 49.00 & 49.00 \\
\hline L25 & -159 & -174.73 & -160.96 & -159.86 & L64 & 49 & 49.00 & 49.00 & 49.00 \\
\hline L26 & -159 & -174.73 & -160.96 & -159.86 & L65 & -282 & -287.30 & -282.66 & -282.29 \\
\hline L27 & -160 & -160.00 & -160.96 & -160.00 & L66 & -282 & -287.30 & -282.66 & -282.29 \\
\hline L28 & 142.7 & 96.57 & 151.81 & 119.96 & L67 & -127 & -173.43 & -118.19 & -150.04 \\
\hline L29 & 50.6 & 75.30 & 51.34 & 50.87 & L68 & -8.8 & -57.41 & 0.75 & 33.90 \\
\hline L30 & 200 & 200.00 & 200.00 & 200.00 & L69 & 200 & 200.00 & 200.00 & 200.00 \\
\hline L31 & -381 & -387.30 & -381.34 & -380.87 & L70 & -439 & -457.94 & -441.19 & -439.86 \\
\hline L32 & -147 & -147.00 & -147.00 & -147.00 & L71 & -439 & -457.94 & -441.19 & -439.86 \\
\hline L33 & -323 & -323.21 & -353.86 & -323.21 & L72 & 189.9 & 193.21 & 190.23 & 189.99 \\
\hline L34 & -323 & -323.21 & -353.86 & -323.21 & L73 & 189.9 & (193.21 & 190.23 & 189.99 \\
\hline L35 & -323 & -323.21 & -353.86 & -323.21 & L74 & 146.7 & 151.12 & 147.22 & 146.91 \\
\hline L36 & 118.8 & 131.29 & 120.13 & 119.24 & L75 & -155 & -155.00 & -155.00 & -155.00 \\
\hline L37 & 118.8 & 131.29 & 120.13 & 119.24 & L76 & -155 & -155.00 & -155.00 & -155.00 \\
\hline L38 & 118.8 & 131.29 & 120.13 & 119.24 & L77 & -82 & -82.00 & -82.00 & -82.00 \\
\hline L39 & -338 & -338.38 & -346.41 & -338.38 & & & & & \\
\hline
\end{tabular}

\section{CONCLUSION}

The evolution of load flow analysis methods has made other power system related studies possible but has also resulted in increased complexity of analysis either at planning, operation or expansion stages. Despite the added complexity on account of increasing network size, this complexity inherently due to the nonlinear nature of the mathematical models of network parameters and their equations. State estimation technique is a viable alternative to AC load flow techniques in estimating network bus parameters from a known state necessary to estimate the line flow of any operating point. Transmission line flow estimates are also possible using DC load flow technique but certain enabling assumptions are the bases of the incompleteness and inexactness of its result when compared with the AC techniques. The

justification to the use of DC load flow for quick estimation of transmission line flows is that its estimates compare proportionally to those from AC load flow so that they are effective when applied for contingency and security analyses. Now, the use of linear distribution factors in estimating transmission line flows from a known operation point yields faster results that match the estimations from DC load flow analysis. These sensitivity factors in the form of PTDF and LODF are calculated and stored for a network and remains valid for use as long as the network is unmodified with the addition of a bus, loads, generators or transmission lines. From any known operation point, the transmission line flow or loading/overload of any other operation point following the outage or variation of generator power 
output or the contingency of a transmission line may be estimated using the stored network values of Power Transfer Distribution Factors (PTDF) and Line Outage Distribution Factors (LODF). Unlike transmission flow results derived from $\mathrm{AC}$ techniques, PTDF and LODF flow estimates are not only non iterative but linear and has the exact value with flows from DC load flow analysis. This work using the Nigerian $330 \mathrm{kV}$ network of 41 bus demonstrates that

1. it is possible to predict transmission line flows besides using load flow analysis or state estimation which are dependent on convergence and telemetry accuracy respectively.

2. it is possible to evaluate the presence and extent of Transmission line overload after the contingency of a single critical network component be it a generator or transmission line.

3. this is a suitable alternate method for quick post contingency analysis in order to screen and rank $\mathrm{N}-1$ (single component) contingencies as against using load flow or continuous load flow methods.

Two recommendations finally are offered for further work. Firstly, it may be observed that the values of PTDF and LODF reported in this work are Slack bus dependent. In other words, the procedure used for PTDF restricts the power transaction from any generator bus to the slack bus while other online generator buses are non participatory. The limitation of this restriction is that at a particular load demand the slack bus or the transmission lines attached to it may be operating at or near its limits and threaten the operation of the system. Using PTDF and LODF base on the participation of all online generators where power variation is distributed to generators based on a defined participation criterion. Secondly, the sensitivity factors of PTDF and LODF used are DC related has evidently has the comparative limitation of the DC load flow analysis technique. A new form of sensitivity factor base on the $\mathrm{AC}$ technique may be defined to estimate quicker results that are more exact to AC load flow result in other to provide alternatives estimate for expanded application like fault analysis. This Thesis focused on sensitivity factors for active power distribution, further studies can redirect focus on reactive power distribution in order to predict voltage stability.

\section{REFERENCES}

1. Peschon J., Piercy D. S., Tinney W. F. and Tveit O. J., "Sensitivity in power systems," IEEE Trans. Power App. Syst., no. 8, pp. 1687-1696, 2007.

2. Wood A. J and Wollenberg B.F.,, "Power Generation, Operation, and Control". John Wiley \& Sons, New York, 2012.

3. Ahmadi $H$. and Lesani $H$, "Transmission congestion management through LMP difference minimization: A renewable energy placement case study," Arab. J. Sci. Eng., vol. 39, no. 3, pp. 1963-1969, Mar. 2014.

4. Marti H., Ahmadi H. and Bashualdo L., "Linear power-flow formulation based on a voltagedependent load model," IEEE Trans. Power Del., vol. 28, no. 3, pp. 1682-1690., 2013

5. Ahmadi H., Martí H., Alsubaie A., "Sensitivity Factors for Distribution Systems. 2013

6. Mazi A. A., Wollenberg B. and Hesse M., "Corrective control of power system flows by line and bus-bar switching," IEEE Trans. Power Syst., vol. 1, no. 3, pp. 258-264, 1989.

7. Rashid, H. A., Afaneen, A. A., \& Mohammed, R. $\mathrm{S}$ : "Simulation of Line outage distribution factors calculation for $\mathrm{N}$ - Buses System". International Journal of Computer Applications. 156, 3, 2016.

8. Chong, S. S., Chang, H. P., Minhan, Y., \& Gilsoo, J. "Implementation of PTDFs and LODFs for Power System Security". Journal of International Council of Electrical Engineering, 1(1), 49-53., 2011.

9. Teoman, G., George, G., \& Minghai, L.: Generalized Line Outage Distribution Factor, IEEE Trans. Power Systems, 27 (2), 879-881, 2007.

10. Singh S. N. and Srivastava S. C., "Improved voltage and reactive power distribution factors for outage studies." IEEE Trans. Power Syst., vol.12, no. 3, pp. 1085-1093, 1997.

11. Khatod D.K, Pant V. and Sharma J. "A novel approach for sensitivity calculations in the radial distribution system," IEEE Trans. Power Del., vol. 21, no. 4, pp. 2048-2057., 2006.

12. Alsac O. and Sttot B. "Fast Decoupled Power Flow" IEEE Trans on Power System, 93, 859869., 1974. 
International Journal of Trend in Scientific Research and Development (IJTSRD) ISSN: 2456-6470

\section{APPENDIX}

Table I: Nigerian Network Generator Data showing output schedules and limits

\begin{tabular}{|c|c|c|c|c|c|c|c|c|c|}
\hline $\begin{array}{c}\text { Generation } \\
\text { Station }\end{array}$ & Bus No & Pg (MW) & Q g & Q max & Q min & Voltage magnitude & MVA & Status & Pmax \\
\hline Kainji & 2 & 600.00 & 0 & 450 & -300 & 1.01 & 100 & 1 & 760 \\
\hline Shiroro & 4 & 275.00 & 0 & 400 & -300 & 1.03 & 100 & 1 & 600 \\
\hline Jebba & 6 & 500.00 & 0 & 400 & -200 & 1.045 & 100 & 1 & 578.4 \\
\hline Olorunsogo & 13 & 400.00 & 0 & 250 & -190 & 1.02 & 100 & 1 & 760 \\
\hline Geregu & 19 & 394.00 & 0 & 300 & -300 & 1.00 & 100 & 1 & 414 \\
\hline Sapele & 20 & 900.00 & 0 & 500 & -500 & 1.00 & 100 & 1 & 1020 \\
\hline Delta & 22 & 710.00 & 0 & 400 & -100 & 1.00 & 100 & 1 & 840 \\
\hline Papalanto & 25 & 304.00 & 0 & 350 & -300 & 1.00 & 100 & 1 & 304 \\
\hline Egbin(slack) & 27 & 900.97 & 0 & 1000 & -1000 & 1.00 & 100 & 1 & 1320 \\
\hline Afam & 29 & 450.00 & 0 & 560 & -400 & 1.00 & 100 & 1 & 702 \\
\hline Alaoji & 30 & 400.00 & 0 & 300 & -100 & 1.00 & 100 & 1 & 1000 \\
\hline Okpai & 31 & 450.00 & 0 & 400 & -100 & 1.045 & 100 & 1 & 480 \\
\hline Mambila & 34 & 130.88 & 0 & 1000 & -1000 & 1.03 & 100 & 1 & 2600 \\
\hline Gurara & 35 & 300.00 & 0 & 260 & -200 & 1.00 & 100 & 1 & 300 \\
\hline Omoku & 37 & 130.00 & 0 & 100 & -100 & 1.02 & 100 & 1 & 150 \\
\hline Calabar & 39 & 490.00 & 0 & 400 & -400 & 1.00 & 100 & 1 & 561 \\
\hline Egbema & 41 & 250.00 & 0 & 320 & -200 & 1.03 & 100 & 1 & 338 \\
\hline
\end{tabular}

Table II: Nigerian Network Bus data showing initial bus voltage magnitude and load schedules

\begin{tabular}{|c|c|c|c|c|c|c|c|c|c|}
\hline Bus Name & Bus No & $\begin{array}{c}\text { Bus } \\
\text { Type }\end{array}$ & Pd (MW) & $\begin{array}{c}\text { Q d } \\
\text { (MVar })\end{array}$ & $\begin{array}{c}\text { Bs } \\
\text { MVar }\end{array}$ & V m & $\begin{array}{c}\text { Base Volt } \\
\text { (KV) }\end{array}$ & V max & V min \\
\hline Kebbi & 1 & 1 & 150 & 60 & 0 & 0.998 & 330 & 1.05 & 0.95 \\
\hline Kainji & 2 & 2 & 0 & 0 & 0 & 1.01 & 330 & 1.05 & 0.95 \\
\hline Jebba & 3 & 1 & 350 & 195 & 0 & 1 & 330 & 1.05 & 0.95 \\
\hline Shiroro & 4 & 2 & 250 & 160 & 0 & 1.03 & 330 & 1.05 & 0.95 \\
\hline Oshogbo & 5 & 1 & 201 & 137 & 480 & 1 & 330 & 1.05 & 0.95 \\
\hline Jebba Gs & 6 & 2 & 0 & 0 & 0 & 1.045 & 330 & 1.05 & 0.95 \\
\hline Katampe & 7 & 1 & 350 & 220 & 0 & 1 & 330 & 1.05 & 0.95 \\
\hline Mando & 8 & 1 & 200 & 125 & 77.83 & 1 & 330 & 1.05 & 0.95 \\
\hline Kumbotso & 9 & 1 & 350 & 220 & 245.40 & 1 & 330 & 1.05 & 0.95 \\
\hline Jos & 10 & 1 & 250 & 125 & 131.79 & 0.979 & 330 & 1.05 & 0.95 \\
\hline Gombe & 11 & 1 & 160 & 95 & 144.38 & 1 & 330 & 1.05 & 0.95 \\
\hline Yola & 12 & 1 & 160 & 90 & 0 & 1 & 330 & 1.05 & 0.95 \\
\hline Olunrunsogo & 13 & 2 & 130 & 70 & 0 & 1.02 & 330 & 1.05 & 0.95 \\
\hline Damaturu & 14 & 1 & 130 & 70 & 0 & 1 & 330 & 1.05 & 0.95 \\
\hline Maiduguri & 15 & 1 & 200 & 150 & 188.59 & 1 & 330 & 1.05 & 0.95 \\
\hline Omotosho & 16 & 1 & 300 & 188 & 254.8 & 1 & 330 & 1.05 & 0.95 \\
\hline Benin & 17 & 1 & 157 & 80 & 77.14 & 1.01 & 330 & 1.05 & 0.95 \\
\hline Ajaokuta & 18 & 1 & 100 & 55 & 0 & 1 & 330 & 1.05 & 0.95 \\
\hline Geregu & 19 & 2 & 0 & 0 & 0 & 1 & 330 & 1.05 & 0.95 \\
\hline Sapele & 20 & 2 & 0 & 0 & 0 & 1 & 330 & 1.05 & 0.95 \\
\hline Onitsha & 21 & 1 & 115 & 42 & 0 & 1 & 330 & 1.05 & 0.95 \\
\hline Delta & 22 & 2 & 0 & 0 & 0 & 1 & 330 & 1.05 & 0.95 \\
\hline Ikeja.west & 23 & 1 & 429 & 248 & 50.161 & & 330 & 1.05 & 0.95 \\
\hline Akangba & 24 & 1 & 470 & 306 & 50.92 & 1.04 & 330 & 1.05 & 0.95 \\
\hline Papalanto & 25 & 2 & 200 & 129 & 0 & 1 & 330 & 1.05 & 0.95 \\
\hline Aja & 26 & 1 & 455 & 286 & 0 & 1 & 330 & 1.05 & 0.95 \\
\hline
\end{tabular}


International Journal of Trend in Scientific Research and Development (IJTSRD) ISSN: 2456-6470

\begin{tabular}{|c|c|c|c|c|c|c|c|c|c|}
\hline Egbim & 27 & 3 & 0 & 0 & 0 & 1 & 330 & 1.05 & 0.95 \\
\hline Aladja & 28 & 1 & 302 & 45 & 0 & 1 & 330 & 1.05 & 0.95 \\
\hline Afam & 29 & 2 & 0 & 0 & 0 & 1 & 330 & 1.05 & 0.95 \\
\hline Alaoji & 30 & 2 & 360 & 218 & 0 & 1 & 330 & 1.05 & 0.95 \\
\hline Okpai & 31 & 2 & 130 & 80 & 0 & 1.045 & 330 & 1.05 & 0.95 \\
\hline New.Heaven & 32 & 1 & 190 & 56 & 0 & 1 & 330 & 1.05 & 0.95 \\
\hline Ayede & 33 & 1 & 139 & 61 & 0 & 1 & 330 & 1.05 & 0.95 \\
\hline Mambilla & 34 & 2 & 130 & 60 & 0 & 1.03 & 330 & 1.05 & 0.95 \\
\hline Guarara & 35 & 2 & 100 & 40 & 0 & 1 & 330 & 1.05 & 0.95 \\
\hline Markurdi & 36 & 1 & 180 & 65 & 0 & 1 & 330 & 1.05 & 0.95 \\
\hline Omoku & 37 & 2 & 185 & 79 & 0 & 1.02 & 330 & 1.05 & 0.95 \\
\hline Ikot Ekpene & 38 & 1 & 140 & 70 & 0 & 1 & 330 & 1.05 & 0.95 \\
\hline Calabar & 39 & 2 & 180 & 56 & 0 & 1 & 330 & 1.05 & 0.95 \\
\hline Owerri & 40 & 1 & 180 & 75 & 0 & 1.03 & 330 & 1.05 & 0.95 \\
\hline Egbema & 41 & 2 & 168 & 86 & 0 & 1 & 330 & 1.05 & 0.95 \\
\hline
\end{tabular}

Table III: Nigerian Network Line Data showing Transmsission Line Parameters

\begin{tabular}{|c|c|c|c|c|c|}
\hline Line-Index & From & To & $\mathbf{R}$ & $\mathbf{X}$ & $\mathbf{B}$ \\
\hline 1 & 1 & 2 & 0.01068 & 0.09246 & 1.2273 \\
\hline 2 & 2 & 3 & 0.00289 & 0.02459 & 0.3178 \\
\hline 3 & 2 & 3 & 0.00289 & 0.02459 & 0.3178 \\
\hline 4 & 3 & 4 & 0.00853 & 0.07330 & 0.9625 \\
\hline 5 & 3 & 4 & 0.00853 & 0.07330 & 0.9625 \\
\hline 6 & 3 & 5 & 0.00557 & 0.04749 & 0.6172 \\
\hline 7 & 3 & 5 & 0.00557 & 0.04749 & 0.6172 \\
\hline 8 & 3 & 5 & 0.00557 & 0.04749 & 0.6172 \\
\hline 9 & 6 & 3 & 0.00029 & 0.00243 & 0.0314 \\
\hline 10 & 6 & 3 & 0.00029 & 0.00243 & 0.0314 \\
\hline 11 & 4 & 7 & 0.00525 & 0.04479 & 0.5816 \\
\hline 12 & 4 & 7 & 0.00525 & 0.04479 & 0.5816 \\
\hline 13 & 4 & 8 & 0.00343 & 0.02913 & 0.3768 \\
\hline 14 & 4 & 8 & 0.00343 & 0.02913 & 0.3768 \\
\hline 15 & 5 & 17 & 0.00877 & 0.07535 & 0.9905 \\
\hline 16 & 5 & 17 & 0.00877 & 0.07535 & 0.9905 \\
\hline 17 & 5 & 17 & 0.00877 & 0.07535 & 0.9905 \\
\hline 18 & 5 & 23 & 0.00880 & 0.07565 & 0.9945 \\
\hline 19 & 5 & 33 & 0.00410 & 0.03486 & 0.4515 \\
\hline 20 & 8 & 9 & 0.00671 & 0.05734 & 0.7477 \\
\hline 21 & 8 & 10 & 0.00695 & 0.05942 & 0.7755 \\
\hline 22 & 8 & 10 & 0.00695 & 0.05942 & 0.7755 \\
\hline 23 & 8 & 10 & 0.00695 & 0.05942 & 0.7755 \\
\hline 24 & 10 & 11 & 0.00923 & 0.07944 & 1.0465 \\
\hline 25 & 10 & 36 & 0.00860 & 0.07389 & 0.9705 \\
\hline 26 & 10 & 36 & 0.00860 & 0.07389 & 0.9705 \\
\hline 27 & 12 & 11 & 0.00923 & 0.07944 & 1.0465 \\
\hline 28 & 13 & 23 & 0.00463 & 0.03941 & 0.5104 \\
\hline 29 & 14 & 11 & 0.00058 & 0.00489 & 0.0631 \\
\hline 30 & 14 & 15 & 0.00431 & 0.03667 & 0.4752 \\
\hline 31 & 14 & 34 & 0.00431 & 0.03667 & 0.4752 \\
\hline 32 & 17 & 18 & 0.00688 & 0.05883 & 0.7676 \\
\hline & & & & & \\
\hline & & & & & \\
\hline 23 & & & & &
\end{tabular}


International Journal of Trend in Scientific Research and Development (IJTSRD) ISSN: 2456-6470

\begin{tabular}{|c|c|c|c|c|c|}
\hline 33 & 17 & 20 & 0.00179 & 0.01519 & 0.1961 \\
\hline 34 & 17 & 20 & 0.00179 & 0.01519 & 0.1961 \\
\hline 35 & 17 & 20 & 0.00179 & 0.01519 & 0.1961 \\
\hline 36 & 17 & 21 & 0.00487 & 0.04149 & 0.5382 \\
\hline 37 & 17 & 21 & 0.00487 & 0.04149 & 0.5382 \\
\hline 38 & 17 & 21 & 0.00487 & 0.04149 & 0.5382 \\
\hline 39 & 17 & 22 & 0.00343 & 913 & 0.3768 \\
\hline 40 & 18 & 17 & 0.00688 & 0.05883 & 0.7676 \\
\hline 41 & 19 & 18 & 18 & 152 & 0.0196 \\
\hline 42 & 19 & 18 & 0.00018 & 0.00152 & 0.0196 \\
\hline 43 & 21 & 30 & 0.00490 & 179 & 0.5422 \\
\hline 44 & 21 & 31 & & 49 & 382 \\
\hline 45 & 21 & 32 & 0.00286 & 429 & 0.3139 \\
\hline 46 & 22 & 28 & & 73 & 0.0000 \\
\hline 47 & 23 & 16 & & 63 & 0.0000 \\
\hline 48 & 23 & 17 & 0.0 & 883 & 0.7676 \\
\hline 49 & 23 & 17 & & 83 & 0.7676 \\
\hline 50 & 23 & 27 & 0.0 & 883 & 0.2432 \\
\hline 51 & 23 & 27 & 0.00 & 383 & 0.2432 \\
\hline 52 & 24 & 23 & 0.00061 & 0.00517 & 0.0667 \\
\hline 53 & 24 & 23 & 61 & 517 & 0.0667 \\
\hline 54 & 25 & 23 & & 47 & 00 \\
\hline 50 & 27 & 26 & 0.00050 & 426 & 0.0549 \\
\hline 56 & 27 & 26 & 0.00050 & 426 & 0.0549 \\
\hline 57 & 28 & 20 & 0.0 & 13 & 0.2471 \\
\hline 58 & 29 & 37 & 18 & 03 & 0.1294 \\
\hline 59 & 29 & 38 & & 29 & 0.3139 \\
\hline 60 & 29 & 38 & 0.0 & 429 & 0.3139 \\
\hline 61 & 30 & 29 & & 89 & 531 \\
\hline 62 & 30 & 29 & 58 & 89 & 0.0631 \\
\hline 63 & 30 & 40 & 0.0 & 731 & 0.3532 \\
\hline 64 & 30 & 40 & & 731 & 0.3532 \\
\hline 65 & 32 & 38 & 86 & 29 & 139 \\
\hline 66 & 32 & 38 & 86 & 429 & 139 \\
\hline 67 & 33 & 13 & & 18 & 0.2356 \\
\hline 68 & 33 & 25 & 0.0 & 322 & 0.2354 \\
\hline 69 & 35 & 8 & 304 & 580 & 0.3336 \\
\hline 70 & 36 & 32 & 0.0 & 0.07447 & 0.9785 \\
\hline 71 & 36 & 32 & 0.0 & 447 & 0.9785 \\
\hline 72 & 36 & 34 & 0.0 & 988 & 1.3325 \\
\hline 73 & 36 & 34 & 0.01150 & 0.09988 & 1.3325 \\
\hline 74 & 37 & 38 & 0.00079 & 0.00669 & 0.0863 \\
\hline 75 & 38 & 39 & 0.00257 & 0.02186 & 0.2825 \\
\hline 76 & 38 & 39 & 0.00257 & 0.02186 & 0.2825 \\
\hline 77 & 40 & 41 & 0.00318 & 0.02701 & 0.3493 \\
\hline
\end{tabular}

